\title{
Disproportionate Emergency Room Use as an Indicator of Community Health
}

\author{
Kelley Bemis*, Samantha Gray, Megan T. Patel and Demian Christiansen \\ Communicable Disease, Cook County Department of Public Health, Forest Park, IL, USA
}

\section{Objective}

To identify geographic clustering of elevated emergency room (ER) usage rates for incorporation into community health assessments (CHA) in suburban Cook County and to validate this metric as a potential sub-county level community health indicator.

\section{Introduction}

Community health assessments are a foundation of public health practice and a prerequisite to achieving public health accreditation. Best practice dictates that CHAs must incorporate qualitative and quantitative data and utilize a number of indicators to create a detailed picture of a community's health. Metrics may describe demographics, social and economic factors, health behaviors, health outcomes, and healthcare access and utilization. Commonly used indicators facilitate cross-jurisdiction comparisons and simplify decisionmaking. However, while many readily available indicators exist on a county level, few have been made available on the sub-county $l_{\text {level }}{ }^{1}$. Syndromic surveillance messages, typically emergency room visit records, contain sub-county level data on patient residence, such as zip code or municipality. As hospitals progress towards meeting Stage 2 Meaningful Use requirements, transmission of syndromic surveillance data to public health entities will become standard. Analysis of emergency room visit data, either in aggregate or by specific syndromes, may be a valuable sub-county level indicator of community health status and access to care that can be standardized across jurisdictions.

\section{Methods}

Emergency room visits in 2014 for suburban Cook County residents were extracted from a syndromic surveillance system, ESSENCE, containing records from 45 hospitals in Northern Illinois. All hospitals in suburban Cook County report to the system. Crude and age-adjusted rates of ER usage by zip code were calculated using population estimates from the 2013 American Community Survey. Rates were further stratified by sex and age. ArcGIS 10.1 was used to visualize and analyze spatial clustering of rates, including identification of hot spots using Getis-Ord Gi* hot spot analysis.

\section{Results}

In 2014, 767,282 residents of suburban Cook County visited emergency rooms reporting to ESSENCE for an age-adjusted rate of 281 visits per 1,000 persons. Rates varied by zip code of residence, with higher rates in the South District. The aggregate rate of zip codes in the South District was 377 visits per 1,000 persons compared to 240 in the North District, 273 in the West District, and 288 in the Southwest District. A similar trend was seen across all age groups and genders, with the exception of persons 65 years and older who exhibited higher rates in the North and Southwest Districts. Hot spot analysis of age-adjusted rates identified a large cluster of 27 zip codes, spanning the South and Southwest Districts, with significantly elevated ER usage (Figure 1, p <.01). Median age-adjusted rate of this cluster was 382 visits per 1,000 persons with a range of 204 to 807. Areas with elevated ER visit rates are consistent with areas of need identified by other community health indicators, such as high infant mortality or low socioeconomic position.

\section{Conclusions}

Spatial analysis of emergency room visit rates by zip code can identify neighborhoods that may have greater public health or medical needs. Syndromic surveillance data has the potential to provide public health departments with a useful and widely available community health indicator at the sub-county level.

\section{Hot Spot Analysis of Age-Adjusted Emergency Room Visit Rates, Suburban Cook County, 2014}

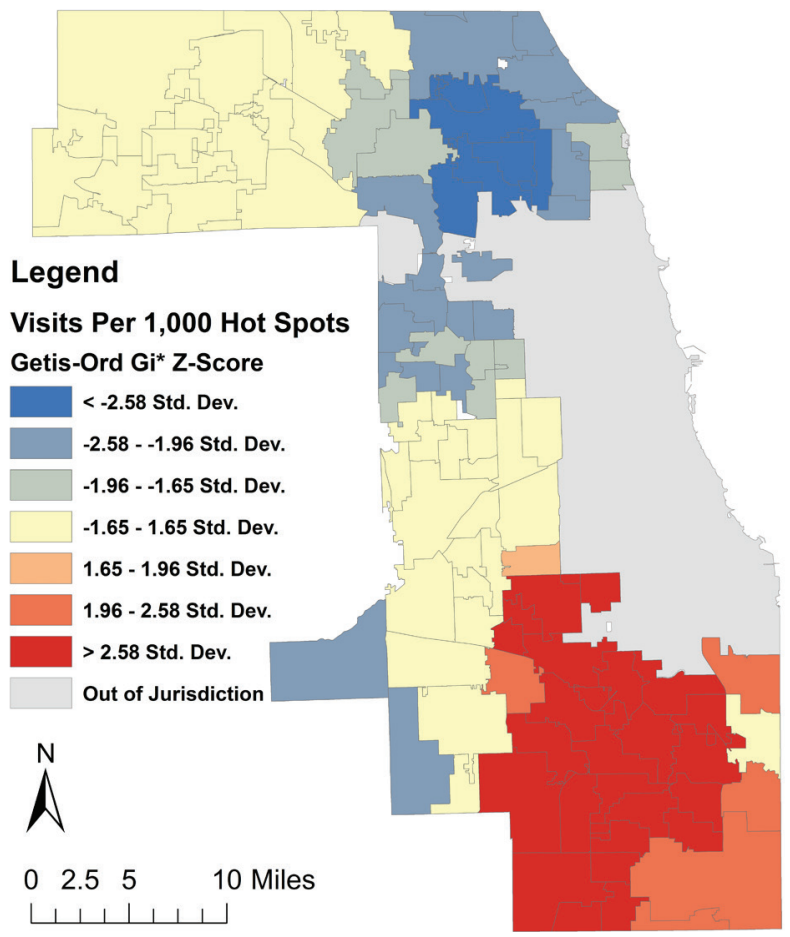

Figure 1. Hot Spot Analysis of Age Adjusted Emergency Room Visit Rates, Suburban Cook County, 2014

\section{Keywords}

Syndromic surveillance; Disparities; GIS; Community health assessment

\section{References}

1. Community Health Assessment for Population Health Improvement: Resource of Most Frequently Recommended Health Outcomes and Determinants. Atlanta, GA: Centers for Disease Control and Prevention; 2013.

\section{*Kelley Bemis}

E-mail: kbemis@cookcountyhhs.org 